(2) Open Access Full Text Article

\title{
Effect and safety of antithrombotic therapies for secondary prevention after acute coronary syndrome: a network meta-analysis
}

This article was published in the following Dove Press journal:

Drug Design, Development and Therapy

\author{
Fanrui Mo' \\ Juan $\mathrm{Li}^{2}$ \\ Yuluan Yan ${ }^{2}$ \\ Weifeng Wu' \\ Shayi Lai \\ 'Department of Cardiology, The \\ First Affiliated Hospital of Guangxi \\ Medical University, Nanning, China; \\ ${ }^{2}$ Department of Cardiology, The \\ Fourth Affiliated Hospital of Guangxi \\ Medical University, Liuzhou, China
}

Correspondence: Weifeng Wu Department of Cardiology, The First Affiliated Hospital of Guangxi Medical University, 6 Shuangyong Road, Nanning 53002I, China

Tel +86 77| 533 I I7।

Fax $+8677 \mid 5359801$

Emailwucna65@163.com
Background: Dual antiplatelet therapy is a standard protocol for secondary prevention after acute coronary syndrome, but despite a variety of new dual antithrombotic strategies, there is a dearth of studies evaluating the effects and safety of some popular therapies. This study used a network meta-analysis to compare the efficacy and safety of all available antithrombotic therapies.

Methods: PubMed, MEDLINE, and Cochrane library databases were searched for randomized controlled trials, published up to July 1, 2017, that evaluated the efficacy of antithrombotic therapy in acute coronary syndrome treatment. The primary endpoints were clinically significant bleeding and major bleeding and secondary endpoints were major cardiovascular events, all-cause deaths, cardiac deaths, and myocardial infarction.

Results: Compared with treatment with aspirin + new P2Y12 inhibitor, treatment with aspirin + new P2Y12 inhibitor converted to clopidogrel clinically reduced the risk of major cardiovascular events or significant bleeding (OR: 0.30, 95\% credibility interval: $0.12-0.75$ ). Both myocardial infarction risk (OR: 0.82, 95\% credibility interval: $0.62-1.09$ ) and major bleeding risk (OR: $0.18,95 \%$ credibility interval: $0.01-1.68$ ) were not significantly different between treatment regimens. There were no significant differences in major cardiovascular events, all-cause deaths, cardiac deaths, myocardial infarction, clinically significant bleeding, and major bleeding risk with rivaroxaban + new P2Y12 inhibitor therapy when compared with aspirin + new P2Y12 inhibitor. Compared with aspirin + clopidogrel, the conversion therapy further reduced the risk of myocardial infarction (OR: 1.81, 95\%, credibility interval: 1.01-1.34) without an increased clinical risk of significant bleeding (OR: 0.41, 95\%, credibility interval: 0.15-1.07). Treatment with aspirin + new P2Y12 inhibitors reduced all-cause deaths (OR: 0.91, 95\% credibility interval: $0.84-0.98$ ) and cardiac death risk (OR: $0.86,95 \%$ credibility interval: $0.79-0.93$ ).

Conclusion: We concluded the following from our study: 1) an aspirin + new P2Y12 inhibitor/ clopidogrel conversion treatment strategy was not inferior to aspirin + new P2Y12 inhibitor; 2) compared with aspirin + clopidogrel, the conversion strategy may further reduce the risk of myocardial infarction without increasing the risk of bleeding; and 3) compared with aspirin + clopidogrel, treatment with aspirin + new P2Y12 inhibitors may result in reduced risk of death.

Keywords: antithrombotic therapies, secondary prevention, acute coronary syndrome, network meta-analysis

\section{Background}

Compared with aspirin combined with clopidogrel, treatment with aspirin combined with new P2Y12 inhibitors, such as prasugrel and ticagrelor, may effectively reduce the incidence of major cardiovascular events (MACE). ${ }^{1,2}$ Consequently, the 2017 BY ${ }_{\mathrm{NC}}$ and incorporate the Creative Commons Attribution - Non Commercial (unported, v3.0) License (http://creativecommons.org/licenses/by-nc/3.0/). By accessing the work you
hereby accept the Terms. Non-commercial uses of the work are permitted without any further permission from Dove Medical Press Limited, provided the work is properly attributed. For permission for commercial use of this work, please see paragraphs 4.2 and 5 of our Terms (https://www.dovepress.com/terms.php). 
European guidelines for revascularization has recommended that aspirin combined with new P2Y12 receptor antagonist is the preferred secondary prevention therapy for acute coronary syndrome (ACS). ${ }^{3}$ However, this treatment program has been associated with an increased risk of bleeding. ${ }^{1,4}$

The TOPIC study divided ACS patients into two groups - the first group was given aspirin orally along with a new P2Y12 receptor antagonist and the second group, after 1 month of oral administration of aspirin, was given clopidogrel instead of new P2Y12 receptor antagonist. ${ }^{5}$ The results showed that this conversion program reduced the incidence of bleeding complications, without an increase in ischemic events, compared with non-conversion. In addition, the ATLAS-ACS 2-TIMI 51 study showed that rivaroxaban, combined with dual antiplatelet therapy, as a triple antiplatelet therapy, reduced MACEs, but with an increased risk of bleeding, compared with conventional dual antiplatelet therapy. ${ }^{6}$ In the GEMINI-ACS-1 study, aspirin was removed from the triple antiplatelet therapy to assess the risk of bleeding for the combination of rivaroxaban with P2Y12 receptor antagonists, resulting in no significant differences in the clinical risk of bleeding, compared with the dual antiplatelet therapy. ${ }^{7}$

Despite the current popularity of a variety of new antithrombotic therapies for the secondary prevention of ACS, there are few clinical randomized controlled trials that compare the safety and efficacy of most of the antithrombotic treatment regimens. Network meta-analysis, as a new methodology, may help to solve this problem. This method indirectly compares two interventions by using a common comparison. For example, if one study compares $\mathrm{A}$ and $\mathrm{B}$, and other study compares $\mathrm{A}$ and $\mathrm{C}$, then $\mathrm{B}$ and $\mathrm{C}$ can be indirectly compared through A. ${ }^{8}$ Finally, our network metaanalysis was based on the combination of direct and indirect comparisons, thereby comparing agents not directly analyzed within an individual trial. ${ }^{8,9}$ In this study, we used the network meta-analysis method to compare the safety and efficacy of the available antithrombotic strategies.

\section{Materials and methods}

\section{Research strategy}

"Acute coronary syndromes", "rivaroxaban", "clopidogrel”, "prasugrel”, "ticagrelor", "aspirin”, “dual antiplatelet”, "new P2Y12 receptor antagonist", "P2Y12 receptor antagonist", and "randomized controlled trial" were used as search terms. We conducted an electronic search of PubMed, MEDLINE, the Cochrane library, and other databases for articles published up to July 1, 2017. Where necessary, a further search of the United States Clinical Trials Database (https://clinicaltrials.gov/29 ${ }^{29}$ was performed for specific information on clinical trials and appropriate data were collected.

\section{Inclusion criteria}

Screening of retrieved articles was carried out according to the following inclusion criteria: 1) studies with a clinical randomized controlled trial study design; 2) studies included patients with ACS; 3) studies that analyzed the efficacy of antithrombotic therapies such as aspirin, aspirin + clopidogrel, aspirin + a new P2Y12 receptor antagonist, aspirin + rivaroxaban, aspirin + clopidogrel + rivaroxaban, rivaroxaban + clopidogrel, rivaroxaban + a new P2Y12 receptor antagonist, aspirin + a new P2Y12 receptor antagonist/clopidogrel, or other studies that compared two or more treatment regimens; and 4) studies that reported on outcomes such as MACEs, all-cause deaths, cardiac deaths, myocardial infarction (MI), major bleeding, and clinically significant bleeding events.

\section{Exclusion criteria}

Study reports that met the following criteria were excluded: 1) observational research, conference literature, and communication articles; 2) studies carried out on patients without ACS; 3 ) studies that compared the efficacy of different doses of intervention drugs, but without a control group; 4) studies that did not include a proposed treatment program; and 5) studies that did not set any outcome events.

\section{Outcomes}

Primary outcomes were major bleeding and clinically significant bleeding events, which were defined according to TIMI and GUSTO criteria, respectively. The secondary outcomes were all-cause deaths, cardiac deaths, MI, and stroke.

\section{Data extraction and quality evaluation}

The data were extracted by two researchers independently. If a study reported results of the same test at different times, or had other forms of repeated reporting, only one test report was included. For controversial decisions on data extraction, the opinion of a third researcher was sought. The data extraction form included the name of the study, the treatment program, the number of events in each group, the total number of subjects, and study characteristics, for example, age, sex, outcomes, and the presence of conditions such as diabetes and hypertension.

For quality assessment of chosen studies, we used the risk assessment tool recommended by the Cochrane Handbook, ${ }^{10}$ with the following guidelines: 1) randomization and its correct application; 2) mention of hidden or covert allocation 
methods and their correct application; 3) use of blinding and its correct application; 4) evidence of complete results; 5) evidence of selective reporting; 6) evidence of other biases, such as funding sources, early termination of research, or other deceptive behaviors.

\section{Statistical methods}

For the traditional meta-analysis, the estimate of effect used for the binary data was OR with a 95\% CI, with the DerSimonianLaird method for statistical analysis. ${ }^{11} I^{2}$ and Cochran Q tests were used to analyze heterogeneity. ${ }^{12} I^{2}<25 \%$ indicated a low degree of heterogeneity; $I^{2}>25 \%$ but $<50 \%$ indicated moderate heterogeneity; and $I^{2}>50 \%$ indicated substantial heterogeneity. STATA 12 software (Stata Corp LP, College Station, TX, USA) was used for meta-analysis.

For the network meta-analysis, the effect was expressed by selection ratio (OR) and $95 \%$ prediction interval (credibility interval; CrI). Due to the unexplained heterogeneity between studies, we used a random effect model for statistical analysis..$^{13}$ We used deviance information criterion (DIC) value to judge model convergence; the smaller the DIC value, the greater the degree of convergence. ${ }^{13}$ The analysis was performed by the Markov Chain Monte Carlo method. ${ }^{13} I^{2}$ was calculated to judge heterogeneity..$^{14} \mathrm{~A}$ consistency analysis was based on the node-splitting model and was judged by the Bayesian $P$-value. ${ }^{14} \mathrm{~A} P$-value $<0.05$ indicated that the direct and indirect comparison results were inconsistent; $P>0.05$ indicated consistency. R software (version 3.2.0), gemtc package, was used for the network meta-analysis. ${ }^{15}$

\section{Results \\ Electronic search results and basic study characteristics}

A total of 4,962 articles were retrieved from electronic searches. After screening, 15 articles were included. ${ }^{1,2,5-7,16-25}$ The literature screening process is shown in Figure 1. A network diagram of all the research scenarios is shown in Figure 2. Basic study characteristics are shown in Table 1.

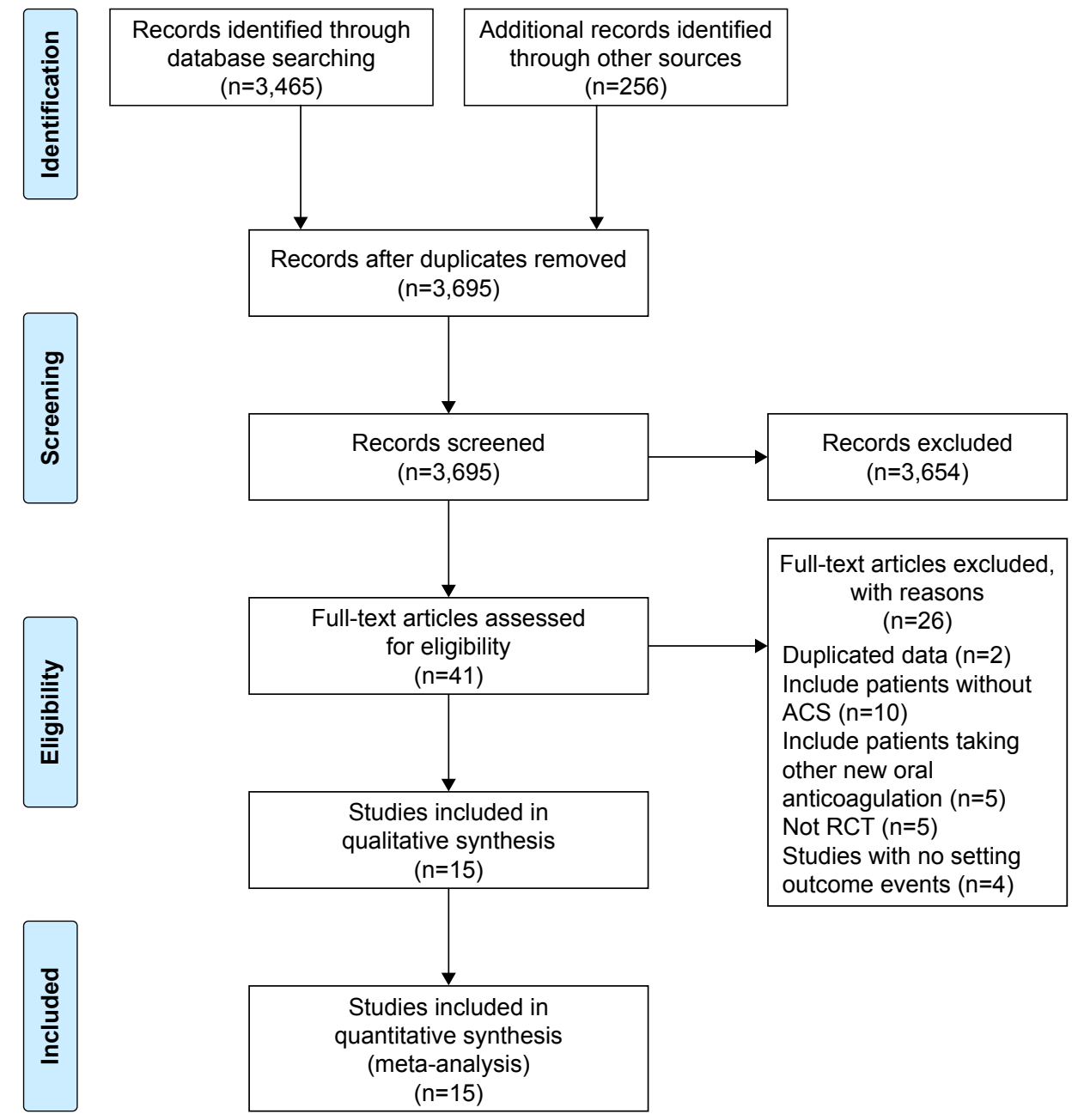

Figure I The flowchart of search results.

Abbreviations: ACS, acute coronary syndrome; RCT, randomized controlled trial. 


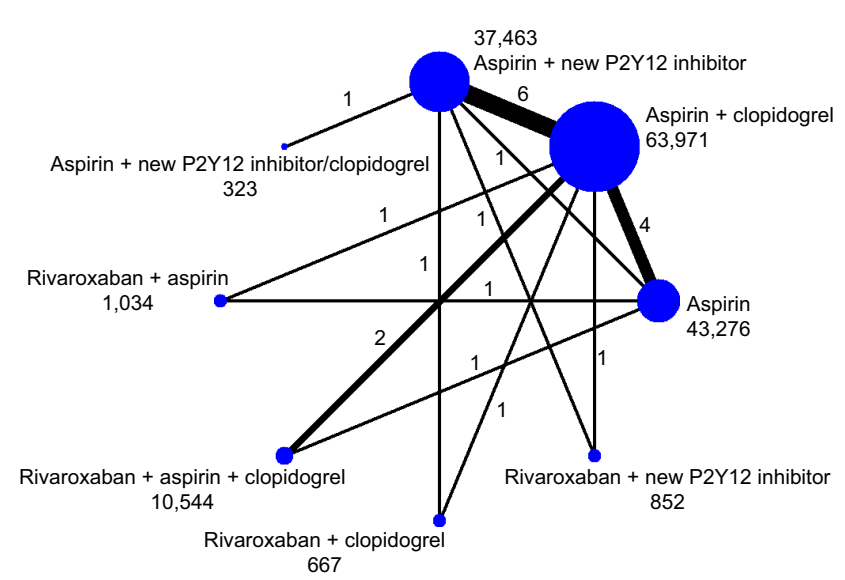

Figure 2 The comparison network for network meta-analysis.

A total of 158,130 patients were included in the analysis. The definition of bleeding was defined according to criteria from TIMI, GUSTO, BARC, PLATO, and clinical practice. The definition of MACE was different among studies. For most studies, MACE was defined as a composite of cardiovascular death, MI, and stroke. The proportion of males ranged from $61 \%$ to $80 \%$, in all studies. The mean age ranged from 57 to 67 years. The proportion of patients with DM ranged from $16 \%$ to $38 \%$. The proportion of patients with hypertension ranged from $43 \%$ to $81 \%$. The proportion of patients with previous MI ranged from 5\% to $43 \%$. The follow-up duration ranged from 1 month to 33 months.

\section{Methodological evaluation of included studies}

All chosen studies were randomized controlled trials and the results of methodological evaluations are shown in Figure 3. Random sequence generation was unclear in two studies..$^{21,24}$ Allocation was unclear in three studies. ${ }^{21,22,24}$ The blinding of participants and personnel was unclear in one study and high risk in another study. ${ }^{5,24}$ Similarly, the blinding of outcome assessment was unclear in one study and high risk in another study. ${ }^{5,24}$ Five studies had other biases. We provided this information in the results section. ${ }^{1,2,20,22,23}$ Overall, the chosen studies were classified as low risk for bias.

\section{Results of clinical outcomes}

\section{Clinically significant bleeding}

Thirteen studies with 147,629 patients were included to evaluate the risk of clinically significant bleeding. ${ }^{1,2,5-7,16-20,22-24}$ The results of network meta-analysis are shown in Table 2.

Compared with aspirin + new P2Y12 receptor antagonist, treatment with aspirin (OR: 0.46, 95\% CrI: 0.31-0.65), aspirin + P2Y12 receptor antagonist (OR: 0.73, 95\% CrI: 0.57-0.93), and aspirin + new P2Y12 receptor antagonist/ clopidogrel (OR: $0.30,95 \%$ CrI: $0.12-0.75)$ significantly reduced the risk of clinically significant bleeding. However, there was no significant difference between rivaroxaban + novel P2Y12 receptor antagonist and aspirin + novel P2Y12 receptor antagonist (OR: 1.28, 95\% CrI: 0.69-2.4).

Compared with aspirin + new P2Y12 receptor antagonist/ clopidogrel, rivaroxaban + aspirin (OR: 3.74, 95\% CrI: 1.19-12.51), rivaroxaban + aspirin + clopidogrel (OR: 9.20, 95\% CrI: 3.17-28), and rivaroxaban + new P2Y12 receptor antagonists (OR: 4.23, 95\% CrI: 1.40-13.21) increased the risk of clinically significant bleeding.

Compared with triple programs, dual programs had a reduced risk of clinically significant bleeding. Of these, aspirin + new P2Y12 receptor antagonist reduced the risk of bleeding (OR: 0.46, 95\% CrI: 0.21-1.02) but the results were not statistically significant.

Compared with rivaroxaban + clopidogrel, treatment with rivaroxaban + new $\mathrm{P} 2 \mathrm{Y} 12$ receptor antagonist resulted in a higher risk of bleeding (OR: 2.16, 95\% CrI: 1-4.76).

\section{Major bleeding}

Fifteen studies with 158,130 patients were included to evaluate the risk of major bleeding. ${ }^{1,2,5-7,16-25}$ The results of network meta-analysis are shown in Table 3.

Compared with aspirin + new P2Y12 inhibitor, therapy with aspirin (OR: 0.64, 95\% CrI: 0.42-0.91) and aspirin + clopidogrel (OR: $0.77,95 \%$ CrI: $0.57-1.02$ ) reduced the risk of major bleeding.

Rivaroxaban + aspirin (OR: 2.63, 95\% CrI: 1-7.02) and rivaroxaban + aspirin + clopidogrel (OR: 4.08, 95\% CrI: 2.03-9.36) increased the risk of major bleeding, while aspirin + new $\mathrm{P} 2 \mathrm{Y} 12$ receptor antagonist/clopidogrel (OR: 0.18, 95\% CrI: 0.01-1.68), rivaroxaban + new P2Y12 receptor antagonist (OR: 1.02, 95\% CrI: 0.27-3.64), and rivaroxaban + clopidogrel (OR: 1.33, 95\% CrI: 0.35-4.74) did not increase the risk of major bleeding.

Compared with aspirin + new P2Y12 receptor antagonist/ clopidogrel, rivaroxaban + aspirin (OR: 14.61, 95\% CrI: 1.27-461.53) and rivaroxaban + aspirin + clopidogrel (OR: 22.9, 95\% CrI: 2.2-09.15) showed an increased risk of bleeding.

Compared with aspirin + clopidogrel, rivaroxaban + aspirin (OR: 3.40, 95\% CrI: 1.33-8.89) and rivaroxaban + aspirin + clopidogrel (OR: 5.27, 95\% CrI: 2.77-11.66) also increased the risk of bleeding. 


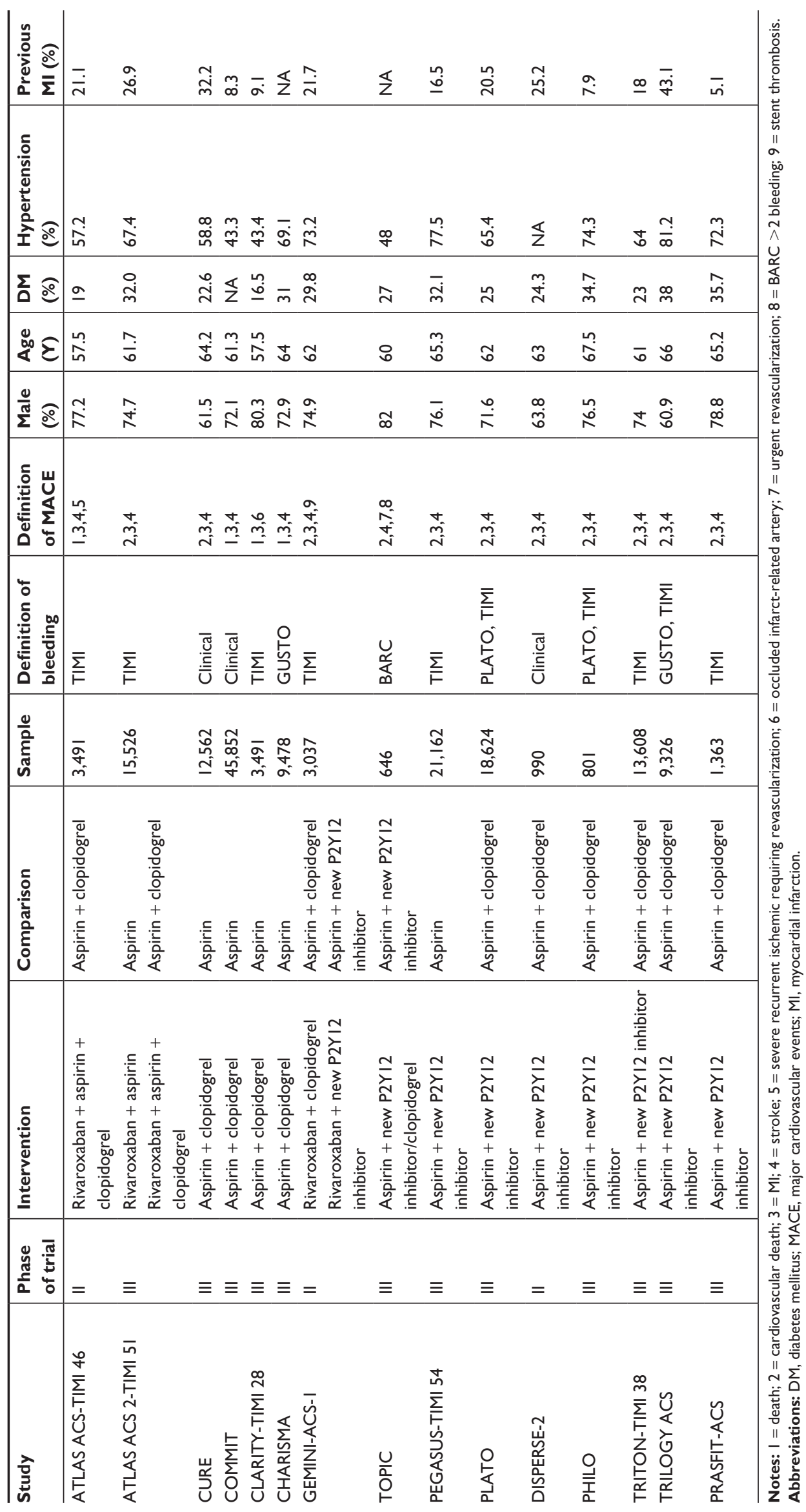




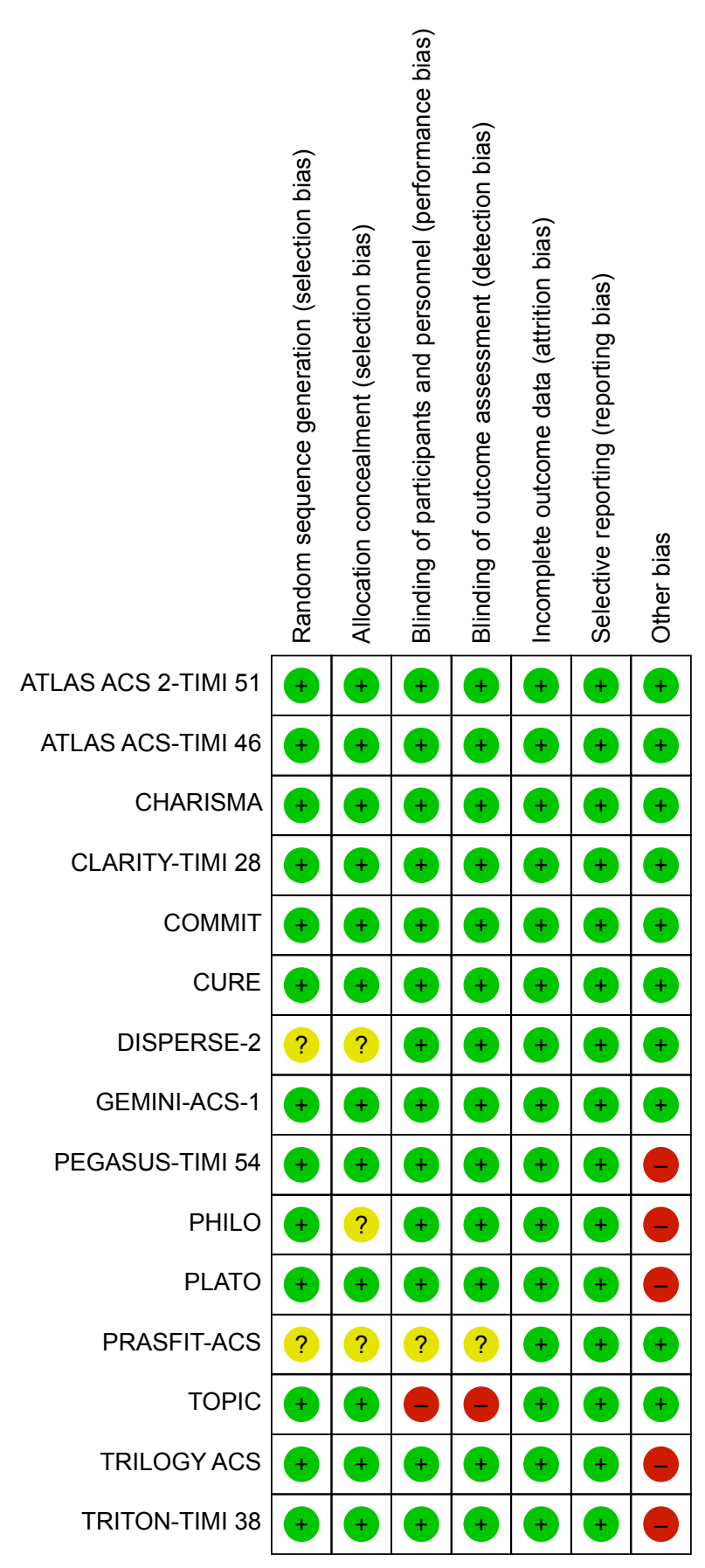

Figure 3 Risk-of-bias assessment of the randomized controlled trials.

\section{Major cardiovascular events}

Fourteen studies with 157,816 patients were included to evaluate the risk of MACE. ${ }^{1,2,5-7,16-20,22-25}$ The results of network meta-analysis are shown in Table 4.

Compared with new P2Y12 receptor antagonist/ clopidogrel therapy, all antithrombotic therapies increased the incidence of MACE except for rivaroxaban + aspirin + clopidogrel therapy (OR: 1.88, 95\% CrI: 0.91-3.75).
There was no significant difference in MACE between rivaroxaban + novel P2Y12 receptor antagonist and aspirin + novel P2Y12 receptor antagonist (OR: 1.02, 95\% CrI: 0.59-1.77).

\section{All-cause deaths}

Twelve studies with 140,294 patients were included to evaluate the risk of all-cause mortality. ${ }^{1,2,7,17-25}$ The results of network meta-analysis are shown in Table 5.

Compared with aspirin, aspirin + clopidogrel (OR: 0.94, 95\% CrI: 0.89-1) and aspirin + new P2Y12 receptor antagonist (OR: 0.86, 95\% CrI: 0.79-0.93) reduced the risk of death from all causes.

Aspirin + new P2Y12 receptor antagonist, in contrast to aspirin + clopidogrel, further reduced the risk of death (OR: 0.91, 95\% CrI: 0.84-0.98). There was no significant difference in all-cause mortality risks between rivaroxaban + novel P2Y12 receptor antagonist and aspirin + novel P2Y12 receptor antagonist (OR: 1.09, 95\% CrI: 0.44-2.68).

\section{Cardiac deaths}

Twelve studies with 95,088 patients were included to evaluate the risk of cardiac deaths. ${ }^{1,2,5,7,17,19-25}$ The results of network meta-analysis are shown in Table 6. Compared with aspirin, aspirin + clopidogrel (OR: 0.92, 95\% CrI: 0.81-1.03) and aspirin + new P2Y12 receptor antagonist (OR: 0.79, 95\% CrI: 0.68-0.91) reduced cardiac deaths.

Compared with aspirin + clopidogrel, aspirin + new P2Y12 receptor antagonist (OR: 0.86, 95\% CrI: 0.79-0.93) further reduced the risk of cardiac death.

There was also no significant difference in the risk of cardiac death between rivaroxaban + novel P2Y12 receptor antagonist and aspirin + novel P2Y12 receptor antagonist (OR: 1.34, 95\% CrI: 0.47-3.85).

\section{Myocardial infarction}

Twelve studies with 131,554 patients were included to evaluate the risk of MI. ${ }^{1,2,5,7,17-19,21-25}$ The results of network meta-analysis are shown in Table 7.

Compared with aspirin + new P2Y12 receptor antagonist, aspirin (OR: 1.48, 95\% CrI: 1.20-0.83) and aspirin + clopidogrel (OR: 1.81, 95\% CrI: 1.01-1.34) increased MI risk, whereas aspirin + new P2Y12 receptor antagonist/ clopidogrel (OR: 0.82, 95\% CrI: 0.62-1.09) and rivaroxaban + novel P2Y12 receptor antagonist (OR: 1.72, 95\% CrI: 0.93-3.17) did not. Compared with aspirin + new P2Y12 receptor antagonist/clopidogrel, treatment with aspirin + clopidogrel (OR: 1.44, 95\% CrI: 1.03-1.94), aspirin (OR: 1.8, 

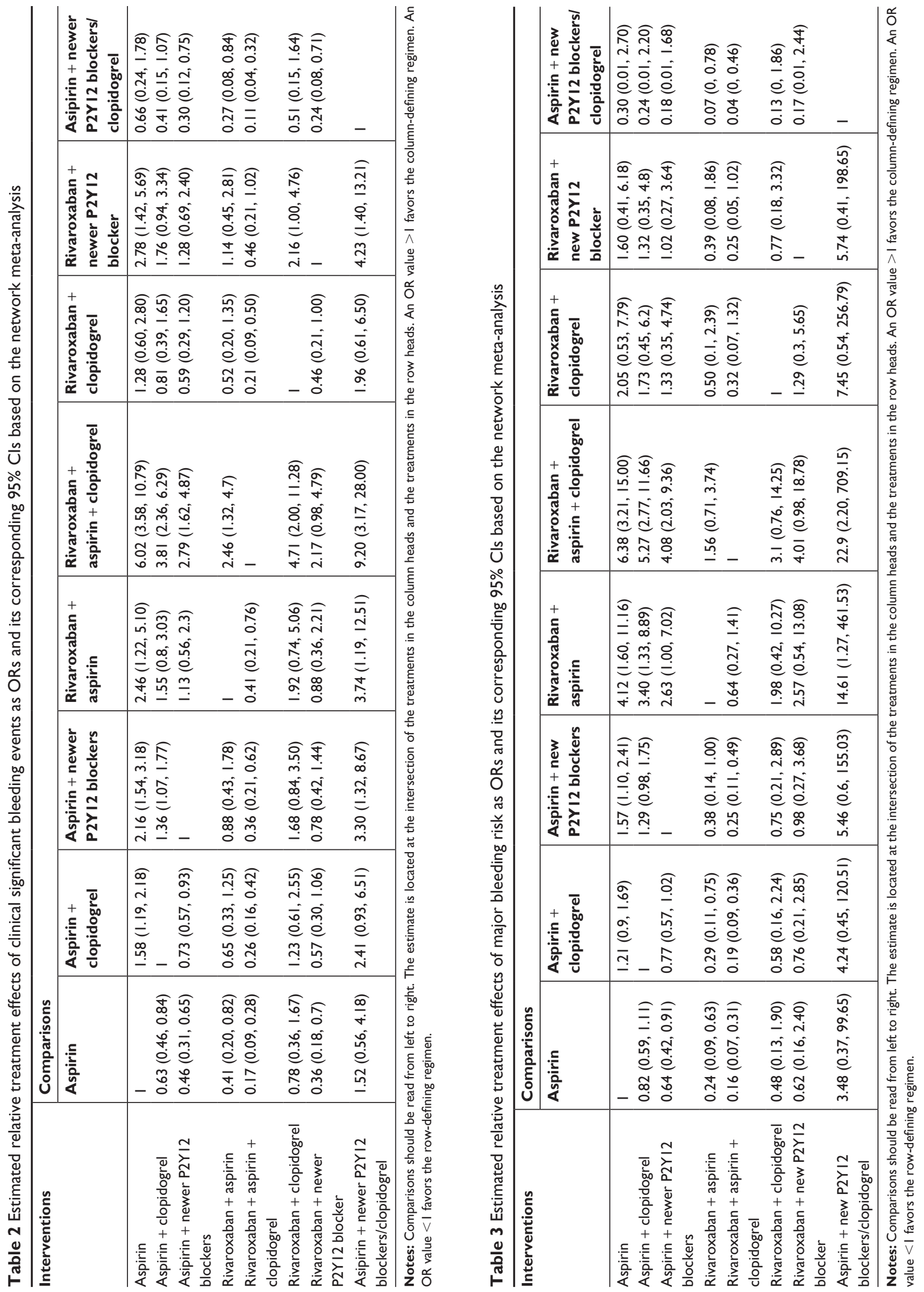


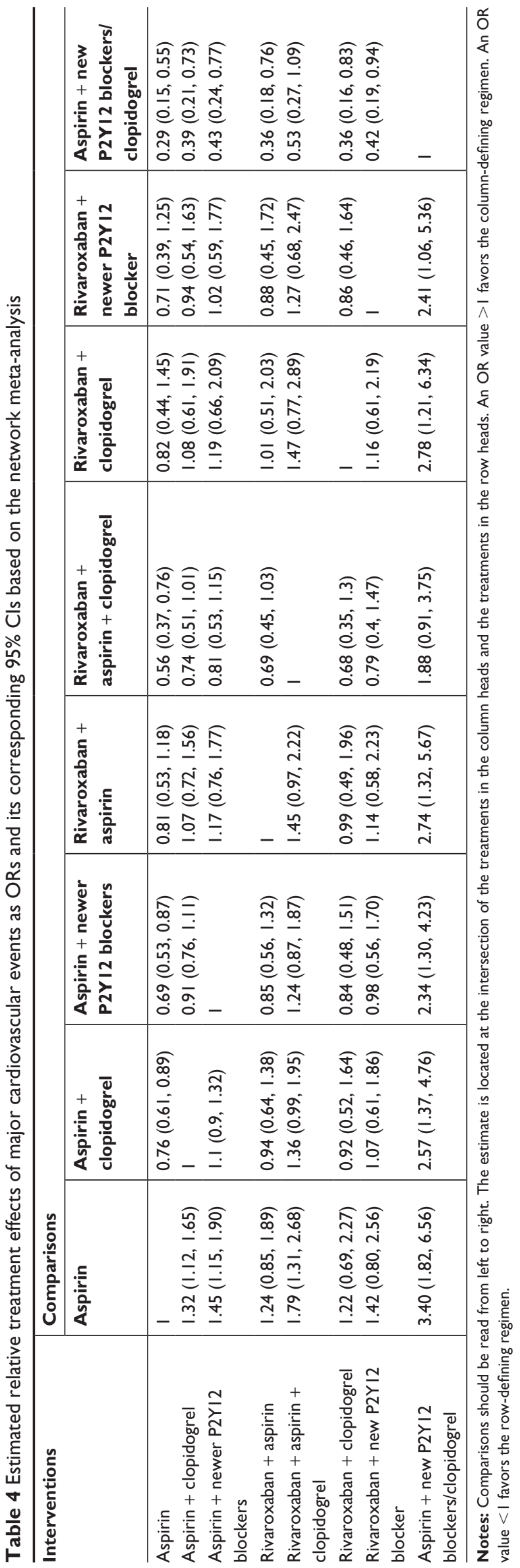

95\% CrI: 1.26-2.55), and rivaroxaban + new P2Y12 receptor antagonist (OR: 2.09, 95\% CrI: 1-4.05) increased MI risk.

\section{Other results}

The convergence degree of each clinical outcome event was better. The node-splitting model demonstrated that the traditional and network meta-analyses results were consistent. For clinically significant bleeding and major bleeding events, $I^{2}$ was $20 \%$ and $60 \%$, respectively, suggesting a lack of substantial heterogeneity. Heterogeneity was $31.2 \%$ for MACE, $23.1 \%$ for all-cause deaths, $35.6 \%$ for cardiac deaths, and $40 \%$ for MI and other events, suggesting low to moderate heterogeneity.

\section{Discussion}

In this study, we used network meta-analysis to evaluate the safety and efficacy of antithrombotic therapies in the treatment of ACS. First, we observed that neither an aspirin + new P2Y12 receptor antagonist/clopidogrel conversion strategy nor treatment with aspirin + new P2Y12 receptor antagonist as a standard dual antiplatelet strategy significantly affected MI incidence or the risk of major bleeding, but the former resulted in reduced MACE incidence and reduced clinically significant bleeding risk.

Second, our results demonstrated that, compared with aspirin + clopidogrel, the conversion strategy could further reduce the risk of MI, without increasing the risk of bleeding.

Third, we found that compared with aspirin + new P2Y12 receptor antagonist, there were no clinically significant benefits from rivaroxaban + new $\mathrm{P} 2 \mathrm{Y} 12$ receptor antagonists in terms of MACE events, all-cause deaths, cardiac deaths, MI, clinically significant bleeding, major bleeding, or any other aspects.

Finally, compared with aspirin + clopidogrel, aspirin + new P2Y12 receptor antagonist reduced the risk of all-cause deaths and cardiac deaths.

ACS patients have unstable plaques that rupture easily, leading to thrombosis, thereby requiring oral antiplatelet agents to reduce the risk of ischemia. The TRITON-TIMI study showed that aspirin combined with prasugrel resulted in significantly reduced incidences of ischemic events compared with aspirin, combined with clopidogrel. ${ }^{2}$ The PLATO trial also demonstrated a net benefit of aspirin combined with ticagrelor in ACS patients. ${ }^{1}$ Subsequently, the ESC/EACTS and ACCF/AHA guidelines recommended aspirin + new P2Y12 receptor antagonist as the preferred antithrombotic therapy in ACS patients. ${ }^{3,26}$ However, in the PLATO study, the ischemic benefit of aspirin + ticagrelor predominantly occurred within 1 month of oral administration and thereafter 
Table 5 Estimated relative treatment effects of death as ORs and its corresponding $95 \%$ Cls based on the network meta-analysis

\begin{tabular}{|c|c|c|c|c|c|}
\hline \multirow[t]{2}{*}{ Interventions } & \multicolumn{5}{|l|}{ Comparisons } \\
\hline & Aspirin & $\begin{array}{l}\text { Aspirin }+ \\
\text { clopidogrel }\end{array}$ & $\begin{array}{l}\text { Aspirin + newer } \\
\text { P2YI } 2 \text { blockers }\end{array}$ & $\begin{array}{l}\text { Rivaroxaban + } \\
\text { clopidogrel }\end{array}$ & $\begin{array}{l}\text { Rivaroxaban }+ \text { new } \\
\text { P2Y I } 2 \text { blocker }\end{array}$ \\
\hline Aspirin & I & $0.94(0.89,1.00)$ & $0.86(0.79,0.93)$ & $0.86(0.38,1.92)$ & $0.93(0.38,2.29)$ \\
\hline Aspirin + clopidogrel & $1.06(1.00,1.12)$ & 1 & $0.91(0.84,0.98)$ & $0.91(0.4 \mathrm{I}, 2.03)$ & $0.99(0.40,2.42)$ \\
\hline $\begin{array}{l}\text { Aspirin + new P2YI2 } \\
\text { blockers }\end{array}$ & $1.17(1.07,1.27)$ & $1.10(1.02,1.19)$ & 1 & $1.00(0.45,2.26)$ & $1.09(0.44,2.68)$ \\
\hline Rivaroxaban + clopidogrel & $\mathrm{I} .17(0.52,2.6 \mathrm{I})$ & $1.10(0.49,2.46)$ & $1.00(0.44,2.25)$ & I & $\mathrm{I} .08(0.32,3.6 \mathrm{I})$ \\
\hline $\begin{array}{l}\text { Rivaroxaban }+ \text { new } \\
\text { P2YI } 2 \text { blocker }\end{array}$ & $1.07(0.44,2.65)$ & I.0I $(0.4 \mathrm{I}, 2.50)$ & $0.92(0.37,2.28)$ & $0.92(0.28,3.09)$ & 1 \\
\hline
\end{tabular}

Notes: Comparisons should be read from left to right. The estimate is located at the intersection of the treatments in the column heads and the treatments in the row heads. An OR value $>$ I favors the column-defining regimen. An OR value $<$ I favors the row-defining regimen.

ischemic benefit reduced and bleeding risk increased. ${ }^{27}$ Thus, whether ticagrelor conversion to clopidogrel after 1 month of ticagrelor increases the risk of bleeding and reduces the risk of ischemia remains inconclusive. The TOPIC study was the only clinical study to address this issue; it included 645 patients and compared the efficacy and safety of conversion and non-conversion strategies. The results showed that the conversion strategy did not increase the risk of ischemia or bleeding compared with the non-conversion strategy. ${ }^{5}$

No studies in our meta-analysis directly compared the conversion strategy with aspirin + clopidogrel therapy. However, we used the network meta-analysis methodology to indirectly compare the efficacy of the two therapies. The results showed that compared with aspirin + clopidogrel, the conversion strategy could further reduce the risk of MI, without increasing the risk of bleeding.

The ATLAS ACS2-TIMI 51 study evaluated the efficacy and safety of rivaroxaban in the treatment of ACS. ${ }^{6}$ The results showed that, compared with dual antiplatelet therapy, patients with ACS treated with a dual platelet therapy plus rivaroxaban had a reduced risk of cardiac death, MI, or stroke, but an increased risk of major bleeding and intracranial hemorrhage.

In the GEMINI-ACS-1 study, for patients receiving the P2Y12 receptor antagonist, the addition of rivaroxaban was not significantly different in terms of clinical bleeding when compared with aspirin. ${ }^{7}$ This conclusion was consistent with our study results. In addition, our study indirectly compared rivaroxaban + new P2Y12 receptor antagonist and aspirin + new P2Y12 receptor antagonist. These results demonstrated no difference in risk for ischemia and bleeding. Because the GEMINI-ACS-1 trial sample size was small and the statistical power insufficient, the effectiveness of rivaroxaban + new P2Y12 receptor antagonists has to be further evaluated.

The CURE study confirmed the important role of aspirin combined with clopidogrel in the treatment of ACS. ${ }^{17}$
Compared with aspirin alone, dual antiplatelet therapy was associated with a further reduction in the composite end points of cardiac death, MI, and stroke. However, when decomposing the composite into individual endpoints, the dual antiplatelet therapy did not reduce the risk of cardiac death. Our study combined direct and indirect evidence to improve statistical efficacy, and the results showed that dual antiplatelet therapy could further reduce the risk of cardiac death compared with aspirin.

The PLATO study showed that aspirin + ticagrelor reduced the risk of cardiac death compared with aspirin + clopidogrel and that the duration continued with the prolongation of medication. ${ }^{1}$ The results of the second PLATO study showed no difference in the therapeutic effect of ticagrelor in genetically resistant and nonresistant populations and that ticagrelor was superior to clopidogrel in gene resistant populations. ${ }^{28}$ Therefore, the survival benefit of ticagrelor, compared with clopidogrel, might be related to the genetic resistance of clopidogrel.

\section{Limitations}

The definitions of MACE differed between studies; therefore, the results for MACE were less credible than the overall efficacy of the dual antiplatelet therapies. A MACE event, as defined by TOPIC, is a composite endpoint of cardiac death, stroke, and bleeding, and the MACE event reduction of the conversion strategy is mainly driven by a reduction in bleeding risk. ${ }^{5}$ The composite endpoint of the PLATO study did not include bleeding events, and oral aspirin + ticagrelor significantly reduced the risk of ischemia compared with aspirin + clopidogrel. ${ }^{2}$ Therefore, to validate whether the conversion strategy significantly reduced ischemia, a bigger sample size and a more rigorous composite endpoint definition are required.

The results from the comparison between aspirin + newer P2Y12 blockers/clopidogrel and aspirin + new P2Y12 

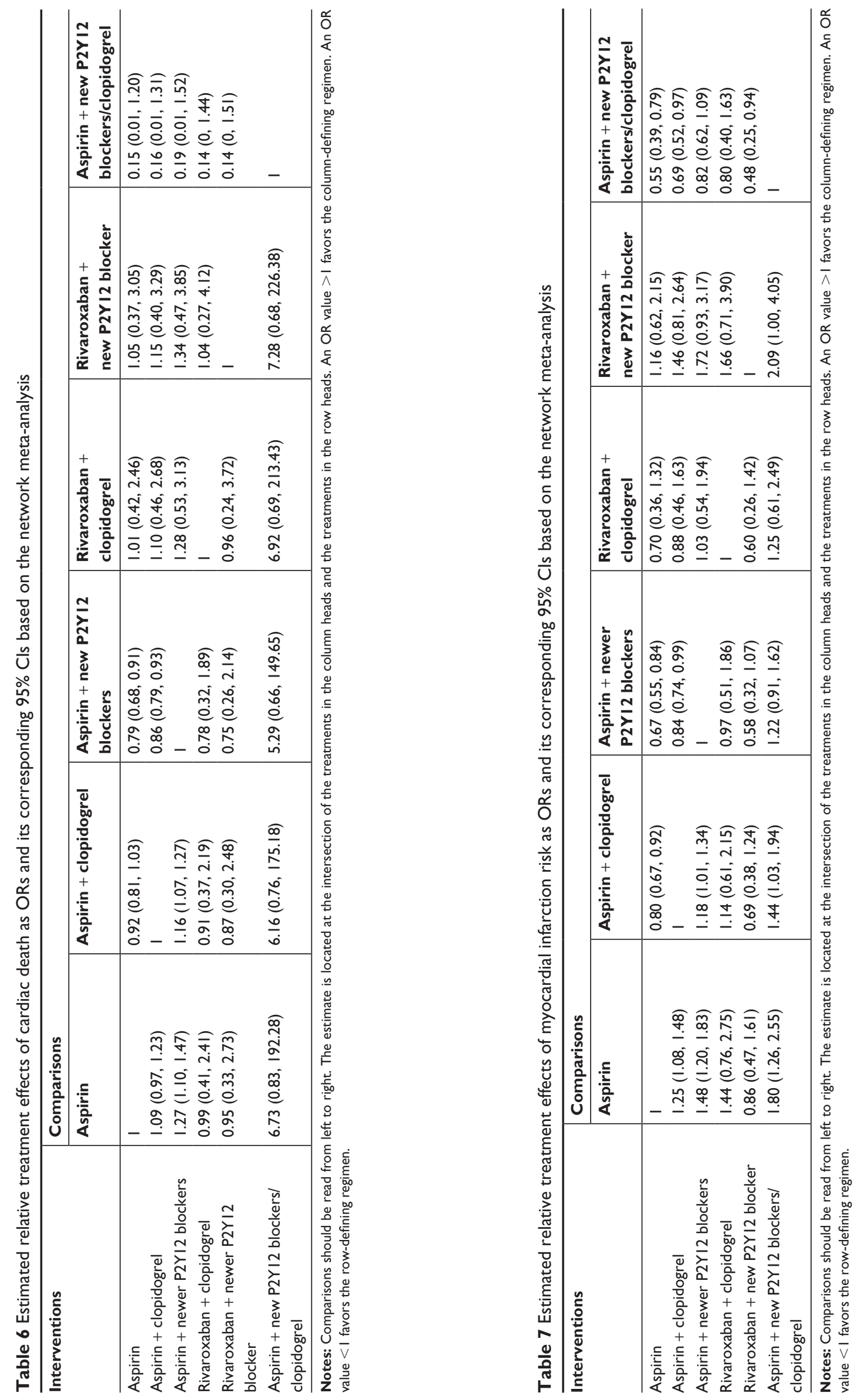
blockers were based mainly on the TOPIC trial, which was the smallest trial included, thanks to the broad CIs. Therefore, further evidence is warranted to evaluate the effectiveness of the conversion therapy.

Some studies did not report death events, for example, the TOPIC trial, and therefore, the conversion strategy could not be compared with other treatment programs for differences in survival. ${ }^{5}$

Our study was based on the study level and individualized data could not be extracted for analysis. Therefore, the best antithrombotic scheme could not be established according to population characteristics.

Stent thrombosis or urgent revascularization events were not reported in many studies. Therefore, we did not evaluate stent thrombosis or urgent revascularization risks in patients taking antithrombotic therapies.

Finally, our study did not consider cost benefits.

\section{Conclusion}

We conclude that 1) the dual antiplatelet therapy of aspirin + new P2Y12 receptor antagonist was not inferior to that of aspirin + new $\mathrm{P} 2 \mathrm{Y} 12$ receptor antagonist/clopidogrel; 2) compared with aspirin + clopidogrel, the conversion strategy may further reduce the risk of MI, without increasing the risk of bleeding; and 3) compared with aspirin + clopidogrel, therapy with aspirin + new P2Y12 receptor antagonists may reduce the risk of death.

\section{Disclosure}

The authors report no conflicts of interest in this work.

\section{References}

1. Wallentin L, Becker RC, Budaj A, et al. Ticagrelor versus clopidogrel in patients with acute coronary syndromes. N Engl J Med. 2009;361(11): 1045-1057.

2. Wiviott SD, Braunwald E, McCabe $\mathrm{CH}$, et al. Prasugrel versus clopidogrel in patients with acute coronary syndromes. N Engl J Med. 2007; 357(20):2001-2015.

3. Valgimigli M, Bueno H, Byrne RA. ESC focused update on dual antiplatelet therapy in coronary artery disease developed in collaboration with EACTS: The task force for dual antiplatelet therapy in coronary artery disease of the European Society of Cardiology (ESC) and of the European Association for Cardio-Thoracic Surgery (EACTS). Eur Heart J. 2018;39(3):213-260.

4. Baber U, Chandrasekhar J, Sartori S, et al. Associations Between Chronic Kidney Disease and Outcomes With Use of Prasugrel Versus Clopidogrel in Patients With Acute Coronary Syndrome Undergoing Percutaneous Coronary Intervention: A Report From the PROMETHEUS Study. JACC Cardiovasc Interv. 2017;10(20):2017-2025.

5. Cuisset T, Deharo P, Quilici J, et al. Benefit of switching dual antiplatelet therapy after acute coronary syndrome: the TOPIC (timing of platelet inhibition after acute coronary syndrome) randomized study. Eur Heart J. 2017;38(41):3070-3078.

6. Mega JL, Braunwald E, Wiviott SD, et al. Rivaroxaban in patients with a recent acute coronary syndrome. N Engl J Med. 2012;366(1):9-19.
7. Ohman EM, Roe MT, Steg PG, et al. Clinically significant bleeding with low-dose rivaroxaban versus aspirin, in addition to P2Y12 inhibition, in acute coronary syndromes (GEMINI-ACS-1): a double-blind, multicentre, randomised trial. Lancet. 2017;389(10081):1799-1808.

8. Glenny AM, Altman DG, Song F, et al. Indirect comparisons of competing interventions. Health Technol Assess. 2005;9(26):1-134.

9. Jansen JP, Crawford B, Bergman G, Stam W. Bayesian meta-analysis of multiple treatment comparisons: an introduction to mixed treatment comparisons. Value Health. 2008;11(5):956-964.

10. Higgins JP, Altman DG, Gøtzsche PC, et al. The Cochrane Collaboration's tool for assessing risk of bias in randomised trials. BMJ. 2011;343:d5928.

11. Dersimonian R, Laird N. Meta-analysis in clinical trials. Control Clin Trials. 1986;7(3):177-188.

12. Higgins JP, Thompson SG, Deeks JJ, Altman DG. Measuring inconsistency in meta-analyses. BMJ. 2003;327(7414):557-560.

13. Dias S, Sutton AJ, Ades AE, Welton NJ. Evidence synthesis for decision making 2: a generalized linear modeling framework for pairwise and network meta-analysis of randomized controlled trials. Med Decis Making. 2013;33(5):607-617.

14. Dias S, Welton NJ, Caldwell DM, Ades AE. Checking consistency in mixed treatment comparison meta-analysis. Stat Med. 2010;29(7-8):932-944.

15. Gertvan V, Joel K. Gemtc: Network meta-analysis using Bayesian methods. R package version 0.8-2. Available from: http://github.com/ gertvv/gemtc. Accessed September 28, 2018.

16. Mega JL, Braunwald E, Mohanavelu S, et al. Rivaroxaban versus placebo in patients with acute coronary syndromes (ATLAS ACS-TIMI 46): a randomised, double-blind, phase II trial. Lancet. 2009;374(9683):29-38.

17. Yusuf S, Zhao F, Mehta SR, et al; Clopidogrel in Unstable Angina to Prevent Recurrent Events Trial Investigators. Effects of clopidogrel in addition to aspirin in patients with acute coronary syndromes without ST-segment elevation. N Engl J Med. 2001;345(7):494-502.

18. Chen ZM, Jiang LX, Chen YP, et al. Addition of clopidogrel to aspirin in 45,852 patients with acute myocardial infarction: randomised placebocontrolled trial. Lancet. 2005;366(9497):1607-1621.

19. Sabatine MS, Cannon CP, Gibson CM, et al. Addition of clopidogrel to aspirin and fibrinolytic therapy for myocardial infarction with STsegment elevation. N Engl J Med. 2005;352(12):1179-1189.

20. Bonaca MP, Bhatt DL, Cohen M, et al. Long-term use of ticagrelor in patients with prior myocardial infarction. $N$ Engl J Med. 2015; 372(19):1791-1800.

21. Cannon CP, Husted S, Harrington RA, et al. Safety, tolerability, and initial efficacy of AZD6140, the first reversible oral adenosine diphosphate receptor antagonist, compared with clopidogrel, in patients with non-ST-segment elevation acute coronary syndrome: primary results of the DISPERSE-2 trial. J Am Coll Cardiol. 2007;50(19):1844-1851.

22. Goto S, Huang CH, Park SJ, Emanuelsson H, Kimura T. Ticagrelor vs. clopidogrel in Japanese, Korean and Taiwanese patients with acute coronary syndrome - randomized, double-blind, phase III PHILO study. Circ J. 2015;79(11):2452-2460.

23. Roe MT, Armstrong PW, Fox KA, et al. Prasugrel versus clopidogrel for acute coronary syndromes without revascularization. NEngl J Med. 2012;367(14):1297-1309.

24. Saito S, Isshiki T, Kimura T, et al. Efficacy and safety of adjusteddose prasugrel compared with clopidogrel in Japanese patients with acute coronary syndrome: the PRASFIT-ACS study. Circ J. 2014;78(7):1684-1692.

25. Bhatt DL, Flather MD, Hacke W, et al. Patients with prior myocardial infarction, stroke, or symptomatic peripheral arterial disease in the CHARISMA trial. J Am Coll Cardiol. 2007;49(19):1982-1988. 
26. Levine GN, Bates ER, Bittl JA. ACC/AHA Guideline Focused Update on Duration of Dual Antiplatelet Therapy in Patients With Coronary Artery Disease: A Report of the American College of Cardiology/American Heart Association Task Force on Clinical Practice Guidelines: An Update of the 2011 ACCF/AHA/SCAI Guideline for Percutaneous Coronary Intervention, $2011 \mathrm{ACCF} / \mathrm{AHA}$ Guideline for Coronary Artery Bypass Graft Surgery, 2012 ACC/AHA/ACP/AATS/PCNA/SCAI/STS Guideline for the Diagnosis and Management of Patients With Stable Ischemic Heart Disease, 2013 ACCF/AHA Guideline for the Management of ST-Elevation Myocardial Infarction, 2014 AHA/ACC Guideline for the Management of Patients With Non-ST-Elevation Acute Coronary Syndromes, and 2014 ACC/AHA Guideline on Perioperative Cardiovascular Evaluation and Management of Patients Undergoing Noncardiac Surgery (vol 68, pg 1082, 2016). J Am Coll Cardiol. 2016;68(10):1150-1151.
27. Becker RC, Bassand JP, Budaj A, et al. Bleeding complications with the P2Y12 receptor antagonists clopidogrel and ticagrelor in the PLATelet inhibition and patient Outcomes (PLATO) trial. Eur Heart J. 2011;32(23):2933-2944.

28. Wallentin L, James S, Storey RF, et al. Effect of CYP2C19 and ABCB1 single nucleotide polymorphisms on outcomes of treatment with ticagrelor versus clopidogrel for acute coronary syndromes: a genetic substudy of the PLATO trial. Lancet. 2010;376(9749):1320-1328.

29. US National Library of Medicine. Available from: https://clinicaltrials. gov/. Accessed August 18, 2017.

\section{Publish your work in this journal}

Drug Design, Development and Therapy is an international, peerreviewed open-access journal that spans the spectrum of drug design and development through to clinical applications. Clinical outcomes, patient safety, and programs for the development and effective, safe, and sustained use of medicines are the features of the journal, which has also been accepted for indexing on PubMed Central. The manuscript management system is completely online and includes a very quick and fair peer-review system, which is all easy to use. Visit http://www.dovepress.com/testimonials.php to read real quotes from published authors.

Submit your manuscript here: http://www.dovepress.com/drug-design-development-and-therapy-journal 